\section{Severe infections in kidney transplanted children}

DOI: $10.1515 / \mathrm{rrlm}-2015-0020$

\section{To the Editor:}

Kidney transplant (KT) represents the ideal therapeutic solution for the child in end stage renal disease (ESRD). Five years survival rate is $94-97 \%$ in case of transplanted patients, compared with $75-87 \%$ in case of children undergoing chronic dialysis. A significant decrease in infection and mortality rate related to infection in kidney transplantation was achieved lately.

In our cohort of 130 transplanted children, the infection incidence rate was $37 \%$, with a mortality rate of $7.7 \%$. The main etiological factors of these infections were represented by cytomegalovirus (CMV) (20\%), urinary tract infections (9.67\%) and fungal infections (15\%). The 5 year survival rate is $88.3 \%$, with a functional graft rate of $70 \%$ at 5 years. Within this report we want to bring to the clinician's attention 2 cases of severe post-transplant infections, focusing on the diagnostic difficulties and treatment options.

Case 1: A female patient, aged 13, with ESRD due to an autosomal recessive polycystic kidney disease, previously diagnosed with Down syndrome, underwent a KT from a living donor (mother). Both patient and donor underwent a biological screening for CMV, Epstein Barr Virus (EBV), hepatitis $\mathrm{B}$ and $\mathrm{C}$ viruses as well as HIV status. Both donor and patient were seronegative for HIV, hepatitis B (HBs Antigen) and $\mathrm{C}$ (IgG HCV). The recipient was $\mathrm{IgG}$ CMV positive, but negative EBV status. The donor was both CMV and EBV positive (IgG titers above the upper limit). The administered immunosuppressive therapy was Cyclosporine (CSA) - mycophenolate mofetil (MMF) - low dose methylprednisolone (MP).

Two months later the patient presented a renal graft dysfunction (Blood urea nitrogen (BUN) $100 \mathrm{mg} / \mathrm{dl}$, creatinine $2.3 \mathrm{mg} / \mathrm{dl}$, metabolic acidosis, oliguria) with no other symptoms. An acute rejection was suspected and MP pulse therapy was started $(20 \mathrm{mg} / \mathrm{kg} / \mathrm{dose})-3$ pulses. BUN and creatinine values returned to normal. A renal biopsy wasn't performed at that time.

Four months after KT the patient presented the same clinical aspect, elevated BUN and creatinine, accompanied by aregenerative anemia, leukopenia $\left(\mathrm{WBC}=3000 / \mathrm{mm}^{3}\right)$ and thrombocytopenia $\left(40000 / \mathrm{mm}^{3}\right)$. A second acute rejection crisis was suspected. To increase the immunosuppression, CSA was changed to Tacrolimus, with a seric level within the therapeutic range. Ancillary tests showed positive CMV IgM antibodies, therefore CMV infection was considered the cause of the acute rejection episode. Consequently a 10 day intravenous Gancyclovir $(5 \mathrm{mg}$ / $\mathrm{kg}$ twice daily) therapy was instituted, followed by oral Gancyclovir prophylaxis.

Despite these treatments the patients clinical state worsened, developing high blood pressure $(180 / 100 \mathrm{mmHg})$ and respiratory distress with a sudden onset. A thoracic CT scan revealed disseminated pulmonary condensation, the patient later dying from a sudden respiratory arrest.

Autopsy revealed disseminated pulmonary condensation areas with right pulmonary artery thrombosis. Histological findings from specimens in the lungs and thrombus were suggestive for pulmonary aspergillosis with extension to the pulmonary artery, endothelial invasion, 
endothelitis and pulmonary artery thrombosis. (Figure 1)

Case 2: Female patient, aged 10, monitored regularly for a KT from a live-donor, due to ESRD with a urological etiology (vesicoureteral reflux nephropathy), returned to our clinic for a routine check. Ancillary tests showed an acute renal graft dysfunction (BUN 130mg/dl; creatinine $3.8 \mathrm{mg} / \mathrm{dl}$ ), despite her normal physical exam and proper diuresis. Her immunosuppressive regimen was $\mathrm{MMF}+\mathrm{CSA}+\mathrm{MP}$.

Renal ultrasound showed a grade V hydronephrosis, with increased resistivity index on Doppler examination. A ureteral anastomosis stenosis with secondary acute kidney injury was suspected. Meantime she developed mild pain in right iliac fossa (graft site). A percutaneous nephrostomy was placed to ensure normal drainage.

The graft dysfunction persisted despite the obstacle by-pass. An ultrasound guided percutaneous renal biopsy was performed, that elicited secondary gross hematuria through the nephrostomy drain. Recombinant coagulation factor VII (Novoseven) was administered, with swift resolution.

Histological findings were suggestive for post-transplant lymphoproliferative disease (PTLD) secondary to EBV infection.

The patient's pre-KT state was EBV IgG and IgM negative. The case was considered as a "de novo" EBV infection following the KT, also confirmed through a serological IgM EBV positive titer.

The immunosuppressive regimen doses were halved and chemotherapy was started at the Oncology department, with poor response followed by infection related death.

The outcome of KT is conditioned by a sum of factors including immunological pathology (acute and chronic rejection), complications of surgery, vascular anastomosis pathology, infectious complications and primary disease recurrence. At present times the risk of post-surgical

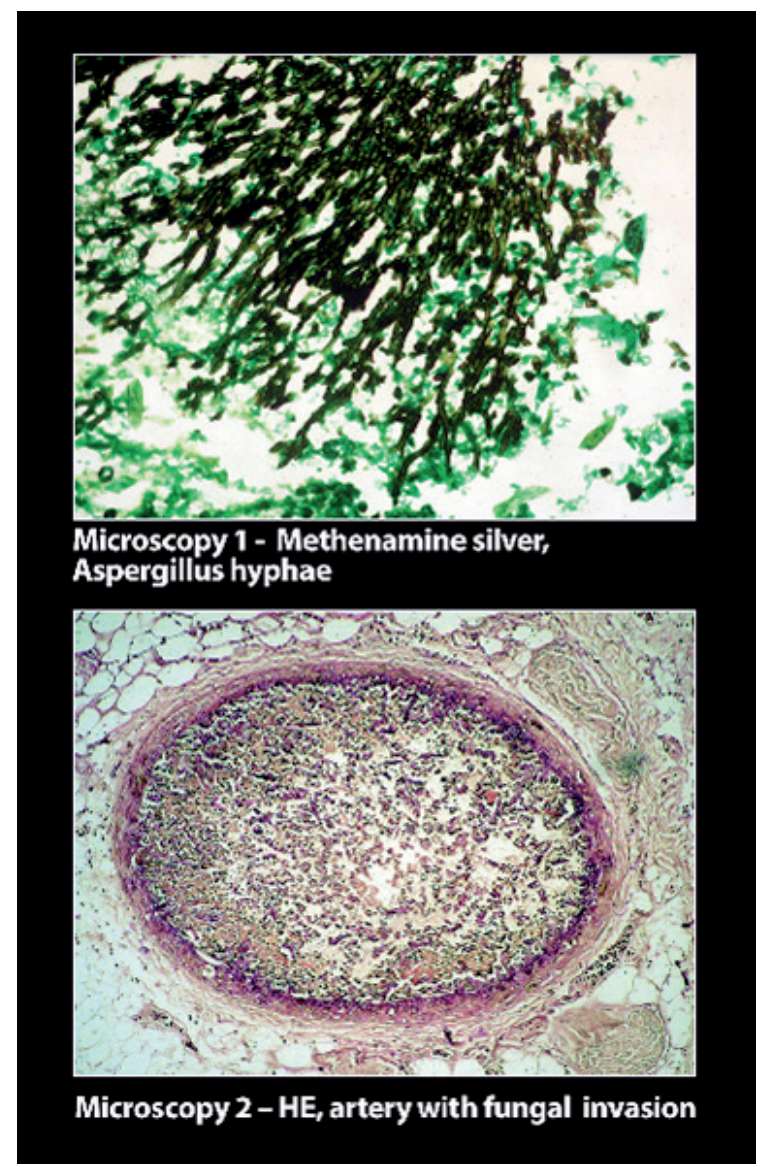

Figure 1. Microscopy images with hematoxylineosine and silver methenamine showing Aspergillus hyphae in lung tissue and artery lumen.

immediate graft failure is uncommon [1], and new immunosuppressive protocols have greatly reduced the risk of immunological acute rejection; chronic rejection and subclinical rejection, as well as allograft nephropathy remain the great challenges of paediatric KT. [2]

Through the use of aggressive treatment protocols for acute rejection, the susceptibility to infection and secondary neoplastic disease were greatly increased, as shown in our 2 case presentations. 
Infection remains the leading cause of hospitalization, following a KT [3].

Bacterial infections are most frequent in the first month after a KT and opportunistic infections occurs mainly in the following 1-6 months period. The following period is associated with community acquired infections, mainly because of the decrease in immunosuppressive needs of the graft. The opportunistic agents most frequently found are immunomodulating viruses such as CMV, EBV, herpes simplex virus, polyomaviruses and fungi such as Aspergillus and Legionella species. The most common of these agents is CMV, therefore most studies concentrating on the prevention and treatment of this infection. The prophylaxis of CMV infection after $\mathrm{KT}$ depends on the donor and recipient CMV status, with the most aggressive ones targeting the donor positive/recipient negative cases $(\mathrm{D}+/$ R-) $[3,4]$.

EBV is another major threat in the 1-6 months post-KT due to its clinical manifestations ranging from mononucleosis syndrome to graft involvement (mimicking acute rejection) [5]. The risk of developing PTLD secondary to EBV infection is greater beyond 6 months post$\mathrm{KT}$. An increase in the occurrence of lymphomas has been documented especially after the introduction of CSA. The situation when the graft recipient is EBV- and the donor is $\mathrm{EBV}+$ poses the greatest risk with regard to the disease's clinical manifestations and subsequently for developing PTLD.

Treatment with anti-T-cell monoclonal antibodies (especially OKT3 acting on CD3 expressing, mature $\mathrm{T}$ lymphocytes) for acute graft rejection and $\mathrm{CMV}+$ status are additional risk factors for PTLD. In the first year post-KT the most common form of PTLD is EBV induced monoclonal proliferation of B cells. Other forms of PTLD, with a more severe outcome, are proliferations of NK-T lymphocytes and null-cell (non-clonal large granular cell lymphocytosis); both are less frequently related to EBV infection [6].

The clinical picture of EBV related PTLD is variable, with extranodal involvement being the most frequent, often with massive lesions in the proximity of the transplanted organ. In PTLD associated with monoclonal proliferation B lymphocytes may infiltrate allografts. Polyclonal variants of PTLD may determine graft infiltrates comprising of both B and T lymphocytes [7].

The diagnosis of our second presented case was a histological "surprise". An acute graft dysfunction accompanied by a urological complication was suspected. The level of clinical suspicion for a possible lymphoproliferative disorder should always remain high, because of the sometimes scarce clinical manifestations of acute EBV infection that can pass unnoticed. Serology for EBV should always be monitored in transplanted patients and PCR assay for EBV viral load can be used to confirm diagnostic suspicion.

Polyclonal proliferations induced by EBV usually respond well to immunosuppressive medication reduction by $50 \%$, further reduction and even withdrawal may be needed, if no response is obtained. Monoclonal variants of PTLD may need administration of anti-CD20 monoclonal antibody (Rituximab) and chemotherapy [8]. In our cohort we had another case that responded well to immunosuppressive dose reduction and Rituximab therapy.

Invasive aspergillosis is less common in KT patients $(<4 \%)$ than in other solid organ transplants $(15 \%)$. Early diagnosis is challenging, with cultures from bronchial lavage lacking sensitivity and the still unclear role of the galactomannan test [9]. In these cases the treatment of choice remains a 12 week course of voriconazole [10].

In our case, the quick development of acute respiratory distress in the absence of previous warning signs raised the suspicion of pulmonary embolism or invasive opportunistic infection. 
CT scan's result was highly indicative of fungal invasion, but unfortunately there was no time to perform a bronchial lavage in order to isolate the etiologic agent. The CMV co-infection is an interesting trait in our case. Even though a correlation between the immunomodulating effect of CMV and Aspergillus infection has not been thoroughly studied, other cases of coinfection have been previously reported [10].

The ongoing battle between graft rejection and infectious complications presents the clinician with a difficult choice when faced with degrading renal function in KT patients. The symptom scarcity at presentation and the possibility of other associated infections or graft complications are added obstacles in the choice of treatment, as shown in the two cases presented. Even more so, we need to maintain a high degree of clinical suspicion, especially in the first 6 months after the transplant.

All KT patients require ongoing close monitoring for subclinical infections and thorough management of immunosuppressive regimens.

\section{Aurel Bizo*, Bogdan Bulata \\ Emergency Clinical Hospital for Children, Cluj Napoca}

* Corresponding author: Aurel Bizo, e-mail: aurelbizo@yahoo.com

\section{Acknowledgements}

Prof. Dr. Petre Florescu, Dr. Dan Gheban; Department of Pathology

Dr. Gheorghita Iacob, Clinical Institute for Urology and Renal Transplant Cluj-Napoca

Received: 27th February 2015; Accepted: $28^{\text {th }}$ May 2015; Published: $7^{\text {th }}$ June 2015

\section{References}

1. Gulati A, Sarwal MM. Pediatric renal transplantation: an overview and update. Curr Opin Pe- diatr. 2010 Apr;22(2):189-96. DOI: 10.1097/ MOP.0b013e32833683fd

2. Lau KK, Giglia L, Chan H, Chan AK. Management of children after renal transplantation: highlights for general pediatricians. Zhongguo Dang Dai Er Ke Za Zhi. 2012 Feb;14(2):81-8.

3. Fernandes A, Rocha L, Costa T, Matos P, Faria M, Marques L et al. Infections Following Kidney Transplant in Children: A Single-Center Study. Open Journal of Nephrology 2014(4):117-24. DOI: $10.4236 /$ ojneph.2014.43017

4. Preiksaitis JK, Brennan DC, Fishman J, Allen U. Canadian Society of Transplantation Consensus Workshop on Cytomegalovirus Management in Solid Organ Transplantation Final Report. Am J Transplant. 2005 Feb;5(2):218-27. DOI: 10.1111/j.16006143.2004.00692.x

5. Cukuranovic J, Ugrenovic S, Jovanovic I, Visnjic M, Stefanovic V. Viral Infection in Renal Transplant Recipients. The Scientific World Journal 2012, Article ID 820621, 18 pages. DOI: 10.1100/2012/820621

6. Yin CC, Medeiros LJ, Abruzzo LV, Jones D, Farhood AI, Thomazy VA.EBV-associated B- and T-cell posttransplant lymphoproliferative disorders following primary EBV infection in a kidney transplant recipient. Am J Clin Pathol. 2005;123(2):222-8. DOI: 10.1309/ PH2BK79HAVTTPW13

7. Swerdlow SH. T-cell and NK-cell posttransplantation lymphoproliferative disorders. Am J Clin Pathol 2007;127:887-95. DOI: 10.1309/LYXN3RGF7D7KPYG0

8. Lee JJ, Lam MS, Rosenberg A. Role of chemotherapy and rituximab for treatment of posttransplant lymphoproliferative disorder in solid organ transplantation. Ann Pharmacother. 2007;41(10):1648-59. DOI: 10.1345/ aph.1K175

9. Walsh TJ, Anaissie EJ, Denning DW, Herbrecht R, Kontoyiannis DP, Marr KA, et al. Treatment of aspergillosis: clinical practice guidelines of the infectious diseases society of America. Clin Infect Dis. 2008;46(3):32760. DOI: $10.1086 / 525258$

10. Estrada C, Desai AG, Chirch LM, Suh H, Seidman R, Darras F, Nord EP. Invasive Aspergillosis in a Renal Transplant Recipient Successfully Treated with Interferon-Gamma. Case Reports in Transplantation 2012;Article ID 493758, 5 pages. DOI: 10.1155/2012/493758 